\title{
Reconocimiento a revisores 2020-2021
}

\author{
Vanesa Pereira Prado ${ }^{1}$ (iD 0000-0001-7747-6718 \\ Carina Patrón ${ }^{1}$ (DD 0000-0002-8662-9437
}

El proceso editorial constituye el eje central mediante el cual se desarrollan las publicaciones de las revistas científicas. Es un proceso complejo que implica la recepción del manuscrito acorde a las normas de la revista, la revisión por pares y la posterior publicación del mismo. La revisión por pares refiere a la evaluación experta de los manuscritos por dos referentes externos, contribuyendo en la decisión editorial sobre la aceptación o rechazo de los mismos. La adecuada elección de los revisores es una tarea esencial para el equipo editorial para, de esta forma, mejorar la calidad de los manuscritos y realizar posibles correcciones inherentes a los mismos.

Durante el proceso editorial es de gran importancia realizar un seguimiento de las etapas en las que se encuentra un manuscrito, así como también mantener una comunicación fluída con los autores y árbitros, de manera de realizar una retroalimentación acerca de las evaluaciones pertinentes.

La aceptación o rechazo de un manuscrito no significa que el mismo sea de mala calidad, sino que muchas veces no sigue la normativa establecida por la revista científica, incumple ciertos requisitos éticos, o simplemente no se considera original ni novedoso ante una temática ampliamente explorada.

Todos los artículos son revisados por dos árbitros del área y si hubiera discrepancia se solicita la opinión de un tercer árbitro. Se tiene la precaución que si un artículo es de autor uruguayo se le asigna árbitros extranjeros y viceversa.

En este período fueron publicados 43 artículos de 65 revisados lo que hace una tasa de rechazo de $33,8 \%$ y de aceptación de 66,3\% (Gráfico 1). Si comparamos con otras revistas del área estamos en un rango similar a las otras publicaciones $(1,2,3)$.

En comparación con el período anterior 2018-2019 la tasa de rechazo fue de 20\% habiendo evaluado 50 artículos (4).

1 Departamento de Publicaciones. Facultad de Odontología. Universidad de la República, Uruguay 


\section{Gráfico 1: Artículos rechazados y aceptados}

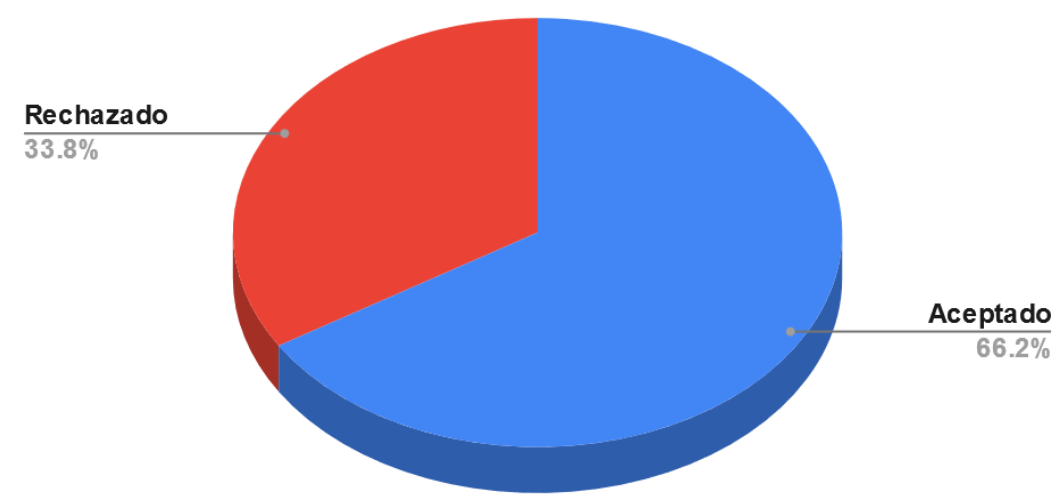

Fueron realizadas 138 revisiones por 97 árbitros de América Latina

Tabla 1: Distribución de revisiones por países

\begin{tabular}{|c|c|}
\hline País & Revisiones realizadas \\
\hline Argentina & 30 \\
\hline Brasil & 9 \\
\hline Chile & 23 \\
\hline Ecuador & 1 \\
\hline México & 5 \\
\hline Panamá & 1 \\
\hline Perú & 1 \\
\hline República Dominicana & 1 \\
\hline Uruguay & 67 \\
\hline Suma total & 138 \\
\hline
\end{tabular}

Tabla 2: Número de árbitros que participaron total

\begin{tabular}{|c|c|}
\hline País & $\mathbf{N}^{\circ}$ \\
\hline Argentina & 22 \\
\hline Brasil & 6 \\
\hline Chile & 17 \\
\hline Ecuador & 1 \\
\hline México & 5 \\
\hline Panamá & 1 \\
\hline Perú & 1 \\
\hline República Dominicana & 1 \\
\hline Uruguay & 43 \\
\hline Suma total & 97 \\
\hline
\end{tabular}

Se puede apreciar en el gráfico 2 que la distribución por género de revisores es muy pareja ya que un 58,2 por ciento son mujeres y un 41,8 por ciento hombres. 
Gráfico 2: Distribución de revisores por género

\section{Distribución de revisores por género}

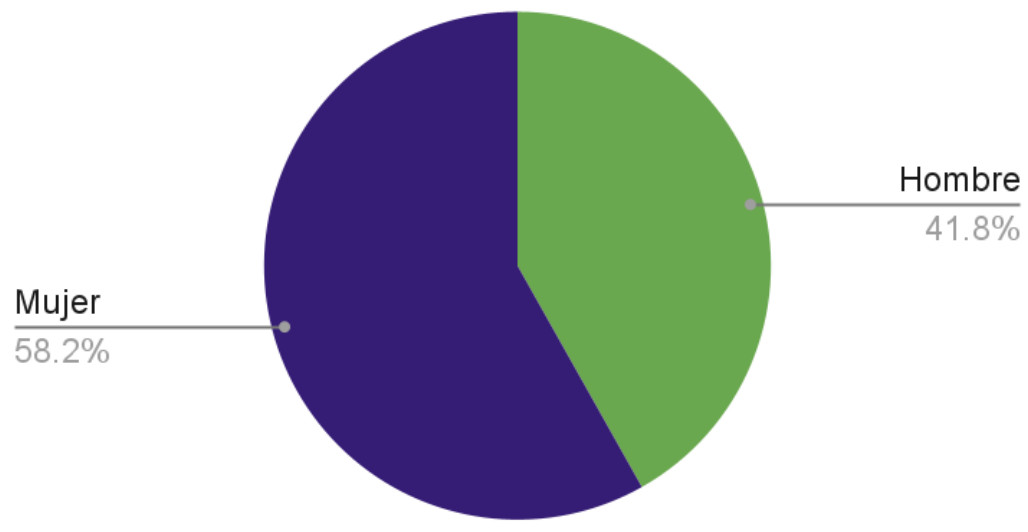

En la siguiente tabla mencionamos y agradecemos a los árbitros que han actuado en los artículos.

Tabla 3: Nombres y países de los revisores 2020-2021

\begin{tabular}{|c|c|}
\hline Revisores & País \\
\hline Actis, Adriana & Argentina \\
\hline Alcántara, Raúl & Chile \\
\hline Álvarez, Licet & Uruguay \\
\hline Andrade, Ernesto & Uruguay \\
\hline Añaña, Nelly & Uruguay \\
\hline Araya, Carlos & Chile \\
\hline Arriagada, María & Chile \\
\hline Arriagada, Verónica & Chile \\
\hline Artaza, Liliana & Argentina \\
\hline Badanian, Andrea & Uruguay \\
\hline Beovide, Verónica & Uruguay \\
\hline Biondi, Ana & Argentina \\
\hline Blanco, Verónica & Uruguay \\
\hline Bologna, Ronell & Uruguay \\
\hline Bonecker, Marcelo & Brasil \\
\hline Borgia, Ernesto & Uruguay \\
\hline Bueno, Luis & Uruguay \\
\hline Cabrera, Carlos & Uruguay \\
\hline Cantero, Cecilia & Chile \\
\hline Casamayou, Martha & Uruguay \\
\hline
\end{tabular}

\begin{tabular}{|c|c|}
\hline Revisores & País \\
\hline Caviglia, Inés & Uruguay \\
\hline Cosetti, Laura & Uruguay \\
\hline Cubillos, Paulina & Chile \\
\hline Cuevas Suárez, Carlos & Brasil \\
\hline Díaz, Alejandro & Chile \\
\hline Dreyer, Erik & Chile \\
\hline Espinosa, Leon & México \\
\hline Fernández, Claudia & Argentina \\
\hline Fierromonti, Claudia & Chile \\
\hline Figueiredo, Marcia Cançado & Brasil \\
\hline Flores, Homero & Chile \\
\hline Fontanetti, Pablo & Argentina \\
\hline Francia, Alejandro & Uruguay \\
\hline Funosas, Esteban & Argentina \\
\hline Galiana, Beatriz & Argentina \\
\hline Garay, Fernando & Uruguay \\
\hline Garchitorena, María Inés & Uruguay \\
\hline García, Andrés & Uruguay \\
\hline García, Graciela & Uruguay \\
\hline Gigena, Pablo & Argentina \\
\hline
\end{tabular}




\begin{tabular}{|c|c|}
\hline Revisores & País \\
\hline Gonzalez, Rogelio & México \\
\hline Grassi, Annabel & Uruguay \\
\hline Grazioli, Guillermo & Uruguay \\
\hline Gugelmeier, Virginia & Uruguay \\
\hline Hermida, Laura & Uruguay \\
\hline Herrero, Cynthia & Argentina \\
\hline Inostroza, Carolina & Chile \\
\hline Jasa, Gainer & Uruguay \\
\hline Kaplan, Andrea & Argentina \\
\hline Kreiner, Marcelo & Uruguay \\
\hline López Jordi, María del Carmen & Uruguay \\
\hline Mandalunis, Patricia & Argentina \\
\hline Martinelli, Sylvia & Uruguay \\
\hline Martins, Manuela & Brasil \\
\hline Masoli, Carla & Argentina \\
\hline Mateu, María Eugenia & Argentina \\
\hline Molgatini, Susana & Argentina \\
\hline Molina, Gustavo & Argentina \\
\hline Moran, María & Chile \\
\hline Moran, María Paz & Chile \\
\hline Muñoz, Helmuth & Chile \\
\hline Obelli, Juan José & Argentina \\
\hline Oliva, Ricardo & México \\
\hline Oyarce, Carmen & Chile \\
\hline Palacios, Silvia & Argentina \\
\hline Pareja, Carmen & Perú \\
\hline Perez Eka & Panama \\
\hline Picca, Mariana & Uruguay \\
\hline
\end{tabular}

\begin{tabular}{|c|c|}
\hline Revisores & País \\
\hline Pisterna, Gabriela & Argentina \\
\hline Puig, Germán & Uruguay \\
\hline Raffo, Marisa & Uruguay \\
\hline Reyes, Ariel & República Dominicana \\
\hline Riva, Raúl & Uruguay \\
\hline Rivas Carlos & Chile \\
\hline Rodríguez, Ismael & Argentina \\
\hline Rodríguez, María Fernanda & Uruguay \\
\hline Romero, René & Uruguay \\
\hline Russo, Ana Laura & Uruguay \\
\hline Sacoto, Fernanda & Ecuador \\
\hline Salveraglio, Inés & Uruguay \\
\hline Samar, María Elena & Argentina \\
\hline Sánchez, Celeste & México \\
\hline Scarrone, Marcelo & Uruguay \\
\hline Sicco, Estefanía & Uruguay \\
\hline Silva, Rhonan & Brasil \\
\hline Silveira, Felipe Martins & Brasil \\
\hline Szwarc, Esther & Uruguay \\
\hline Taramaso, Fernando & Uruguay \\
\hline Valenzuela, Cecilia & Uruguay \\
\hline Valez, Valeria & Uruguay \\
\hline Vázquez, Susana & Uruguay \\
\hline Verdera, Sergio & Uruguay \\
\hline Villanueva, Francisco & México \\
\hline Vuoto, Elena & Argentina \\
\hline Wendler, Michael & Chile \\
\hline Zepedeo, Martín & Uruguay \\
\hline
\end{tabular}

\section{Referencias}

1. Acta Odontológica Colombiana. Instrucciones a los autores. Disponible en: Sobre la revista | Acta Odontológica Colombiana (unal.edu.co)

2. Revista KIRU. Instrucciones a los autores. Disponible en:https://www.aulavirtualusmp.pe/ojs/index. $\mathrm{php} /$ Rev-Kiru0/about/editorialPolicies

3. Revista de Odontopediatría Latinoamericana. Instrucciones a los autores. Disponible en: https://www. revistaodontopediatria.org/index.php/alop/information/authors

4. Lopez Jordi MC, Piovesan S, Pereira V, Patrón C, Lima A. Proceso de arbitraje para la calidad de las revistas científicas. Odontoestomatología 2019; 21(34). Disponible en: http://www.scielo.edu.uy/scielo. php?script=sci_arttext\&pid=S1688-93392019000200074\&lng=es\&nrm=iso\&tlng=es 